\title{
Buckling analysis of rod structure of the two-tier dome
}

\author{
Alexey Beskopylny ${ }^{1, *}$, Alexander Zhuravlev ${ }^{1}$, and Alexander Shilov ${ }^{1}$ \\ ${ }^{1}$ Don State Technical University, pl. Gagarina, 1, Rostov-on-Don, 344010, Russia
}

\begin{abstract}
The paper deals with the problem of calculating the rod structure of a two-tier dome for stability. Such structures consisting of straight rod elements are widely used in practice and have a number of significant advantages. First of all, it is a high rigidity with a low mass. Also, this type of construction is well resistant to dynamic loads. At the same time, the dome model allows for the bifurcation of points on the equilibrium state curve of the system. The problem of the dynamic stability of double dome is solved by the principle of stationarity of total potential energy of the system. The analysis of the obtained solution is carried out, and the equilibrium state diagram for a two-tier core dome is constructed.
\end{abstract}

\section{Introduction}

Spatial rod structures in the form of shells of rotation consist of straight prismatic elements connected at nodes and belonging to a curved surface. Their main advantage is their small self-weight combined with great rigidity. Due to the fact that the share of variable load on the core system, as a rule, prevails in its total intensity, the question arises: can the structure withstand this load distribution during operation? In the presence of initial imperfections, the core structure has a characteristic feature of the abrupt transition from the stable equilibrium to the deformed state with a large amount of nodal displacements.

A significant role is played by the characteristic of the degree of flatness of the considered rod structure of the two-tier dome, which is estimated by the ratio of the diameter of the horizontal circle of its base to the height. It is known that for $\mathrm{d} / \mathrm{h} \leq 8$ the dome model allows for the case of bifurcation of points on the equilibrium state curve of the system $[1,2]$.

The results of a numerical and experimental investigation into static stability of externally pressurized hemispherical and tori spherical domes are considered in [3, 4]. It revealed many difficulties which exist when dealing with layered metallic shells [5].

The numerical approach is widely spread in buckling analysis. The behavior of the stability of the egg-shaped domes was experimentally and numerically analyzed in [6]. Numerical buckling analysis of the egg-shaped dome with perfect geometry and a massequivalent hemisphere was examined. In [7] aluminum sandwich structure with the folded square dome as a core is considered numerically. Testing to a failure of a shallow thin-walled dome made from composite concrete under uniform internal pressure is analyzed in [8].

\footnotetext{
*Corresponding author: besk-an@ya.ru
} 
Spherical domes are widely used as a roof structure on large buildings: buckling normally controls the design. The paper [9] presents the study of the influence of realistic boundary conditions, in the form of a ring at the eaves, on the linear bifurcation of these domes.

The buckling analysis relates to geometrically nonlinear problems and to solve differential equations often use Bubnov-Galerkin methods [10, 11], genetic algorithms [12] and practices based on Fourier transforms [13, 14, 15].

Thus, the development of new approaches for engineering calculations of dome structures is relevant, but before proceeding to the study of the stability of rod structures in the form of convex polyhedra, let us find out the conditions that can correspond to the beginning of the branching points of the deformation curves for specific models of spatial configurations.

Since the icosahedron is usually chosen as the initial polyhedron for the construction of structural networks on a sphere, it is quite elementary, but visual reasoning about the flatness of the rod structure allocated on its surface in the form of a pentagonal pyramid shows that in this case, the ratio of the diameter of the horizontal circle to the height of the pyramid element is $d / h=\sqrt{2(3+\sqrt{5})} \approx 3,24$.

\section{Materials and methods}

The problem is solved by the method of minimizing the functional of the total potential energy of deformation.

For the selected part of the core icosahedron $d / h \leq 8$, it can be argued that there is always a situation associated with the change of equilibrium forms. This equally applies to such a configuration as the 80 -fringe. For this reason, we choose two following options for crushing the structural mesh on the sphere, namely: rod structures in the form of two-tier parts 180 and 320-fringe (Fig.1).
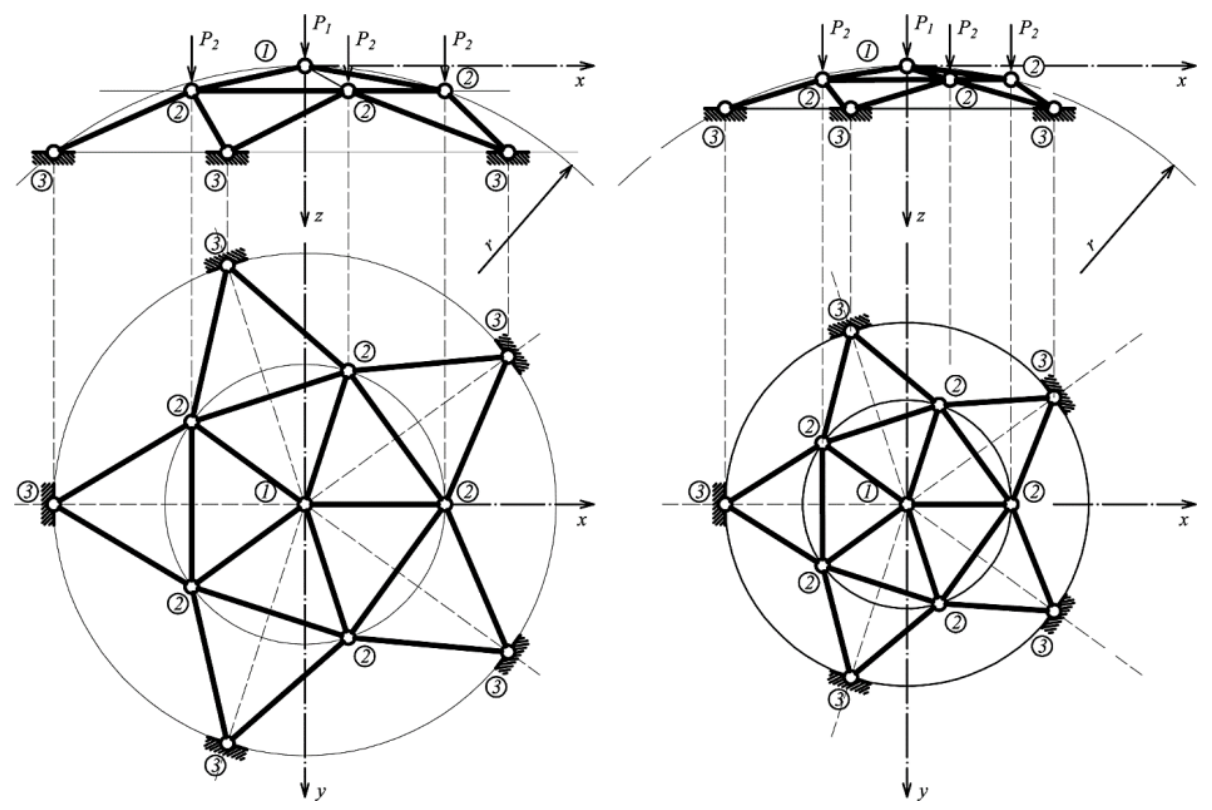

Fig. 1. The rod structure of the two-tires dome: a) in the form of 180-fringes; b) in the form of 360fringes 
Let both spatial point lattices belong to spherical surfaces of the same radius r. For greater generality, suppose that the geometric construction of structural networks on a sphere uses the method of central projection, in which the edges of the icosahedron are divided into three or four segments of equal length. The points of division of the icosahedron edges obtained in this way are connected to each other, as a result of which each of its twenty faces is divided into nine or, respectively, into sixteen equilateral triangles. Then all the nodal points are projected on the surface of the sphere from its center, which eventually leads to the construction of structural networks of core polyhedra.

The radius of the horizontal circle at the base of the two-tier part of the 180-granary is

$$
r_{0}=\sqrt{\frac{2(5-\sqrt{5})}{15}} \approx 0,67062 r
$$

In turn, the application of the top of the pentagonal pyramid of the considered polyhedral configuration is

$$
h=r\left(1-\sqrt{\frac{5+2 \sqrt{5}}{15}}\right) \approx 0.205346 r
$$

It can be seen that $d / h=5.9 \leq 8$ and in this regard here the determining factor will be the work of rod elements exclusively on the longitudinal bend. Therefore, the study of dynamic stability is advisable to carry out only for the rod system in the form of a two-tier part of the 320-granary, for which the height of the rise, expressed in fractions of the radius of the described near it sphere, will be

$$
h_{2}=\frac{5-2 \sqrt{5}}{5} r \approx 0.105573 r
$$

In turn, the radius of the horizontal circle at the base of the rod system is determined by the expression

$$
r_{0,2}=\frac{\sqrt{5}}{5} r \approx 0.447214 r
$$

The diameter of the base of the rod structure and its height are obtained in the latter case equal to

$$
d=2 \sqrt{5} r / 5 ; \quad h=(5-2 \sqrt{5}) r / 5, \text { and } \frac{d}{h}=2(2+\sqrt{5})=8.47 .
$$

For the two-tier of 320-fringes in the equilibrium states of the system should allow for the possibility of the appearance of a limit, and forked points on the diagrams of deformation of the rod design. At the same time, of course, the form of the unstable state will depend on the nature of the distribution of the nodal load. At the same time, it can be shown that for the pyramidal element of the rod structure located within the upper tier of the 320-fringe, $h / d=$ $15.67 \geq 10$, which is a direct indication of the high sensitivity of the system to the central node to bifurcation snap on the vertical axis of symmetry.

The system under consideration in the form of a convex polyhedron is a spatial farm. It has hinged joints in all nodes and is loaded with six vertical forces: in the central node by force $P_{1}$ and in five peripheral nodes of the pentagonal ring by forces $P_{2}$. The stiffness of all 20 truss rods is considered to be the same, and their lengths, expressed in terms of the radius described near the core polyhedron of the sphere, are taken to be equal 


$$
\begin{gathered}
l_{12}=r \sqrt{2\left(1-\sqrt{\frac{23+3 \sqrt{5}}{25+3 \sqrt{5}}}\right)} \approx 0.253185 r, \\
l_{22}=r \sqrt{\frac{7-2 \sqrt{5}}{29}} \approx 0.295242 r, \\
l_{23}=r \sqrt{2-\sqrt{\frac{163+63 \sqrt{5}}{45+17 \sqrt{5}}}} \approx 0.294531 r .
\end{gathered}
$$

As you can see, the difference in the lengths of rods 2-2 and 2-3 is very small and it is possible to take $l_{22} \approx l_{23}$.

\section{Results and discussion}

The total energy of the elastic system is recorded as

$$
\mathbf{E}=\mathbf{U}+\mathbf{V}=\frac{5}{2} E F\left(\frac{\varepsilon_{12}^{2}}{l_{12}}+\frac{\varepsilon_{22}^{2}}{l_{22}}+2 \frac{\varepsilon_{23}^{2}}{l_{23}}\right)-P_{1} w_{1}-5 P_{2} w_{2} .
$$

Deformations in inclined bars are determined by the expression

$$
\begin{gathered}
\varepsilon_{i j}=\left(u_{j}-u_{i}\right) \cos \alpha_{i j}+\left(v_{j}-v_{i}\right) \cos \beta_{i j}+\left(w_{j}-w_{i}\right) \cos \gamma_{i j}+ \\
+\frac{1}{2} l_{i j}\left[\left(\frac{u_{j}-u_{i}}{l_{i j}}\right)^{2}+\left(\frac{v_{j}-v_{i}}{l_{i j}}\right)^{2}+\left(\frac{w_{j}-w_{i}}{l_{i j}}\right)^{2}\right]
\end{gathered}
$$

In accordance with this, we obtain

$$
\begin{aligned}
& \varepsilon_{12}=u_{2} \cos \alpha_{12}+\left(w_{2}-w_{1}\right) \cos \gamma_{12}+\frac{1}{2} l_{12}\left[\left(\frac{u_{2}}{l_{12}}\right)^{2}+\left(\frac{w_{2}-w_{1}}{l_{12}}\right)^{2}\right], \\
& \varepsilon_{23}=-u_{2} \cos \alpha_{23}-w_{2} \cos \gamma_{23}+\frac{1}{2} l_{23}\left[\left(\frac{u_{2}}{l_{23}}\right)^{2}+\left(\frac{w_{2}}{l_{23}}\right)^{2}\right] .
\end{aligned}
$$

To determine $\varepsilon_{22}$ can serve as a simple ratio of the form

$$
\varepsilon_{22}=\sqrt{\frac{5-\sqrt{5}}{2}} u_{2}
$$

Given this relationship, which is linear, nonlinear terms $\left(\frac{u_{2}}{l_{12}}\right)^{2}$ and $\left(\frac{u_{2}}{l_{23}}\right)^{2}$ in expressions (3) and (4) can be neglected as values of higher order of smallness.

The potential strain energy is written as follows

$$
\begin{gathered}
\mathbf{U}=\frac{5}{2} E F l_{12}\left\{\xi_{2}^{2} \cos ^{2} \alpha_{12}+\left(\eta_{2}-\eta_{1}\right)^{2} \cos ^{2} \gamma_{12}+\frac{1}{4}\left(\eta_{2}-\eta_{1}\right)^{4}+\right. \\
+2 \xi_{2}\left(\eta_{2}-\eta_{1}\right) \cos \alpha_{12} \cos \gamma_{12}+\xi_{2}\left(\eta_{2}-\eta_{1}\right)^{2} \cos \alpha_{12}+\left(\eta_{2}-\eta_{1}\right)^{3} \cos \gamma_{12}+
\end{gathered}
$$




$$
\begin{gathered}
+2 m\left[\xi_{2}^{2} \cos ^{2} \alpha_{23}+\eta_{2}^{2} \cos ^{2} \gamma_{23}+\frac{1}{4} m^{2} \eta_{2}^{4}+2 \xi_{2} \eta_{2} \cos \alpha_{23} \cos \gamma_{23}-\right. \\
\left.\left.-m \xi_{2} \eta_{2}^{2} \cos \alpha_{23}-m \eta_{2}^{3} \cos \gamma_{23}\right]+n \xi_{2}^{2}\right\}
\end{gathered}
$$

Where $\xi_{2}=u_{2} / l_{12}, \eta_{1}=w_{1} / l_{12}, \eta_{2}=w_{2} / l_{12}$, are dimensionless values of displacement; and $m=\frac{l_{12}}{l_{22}}, n=(5-\sqrt{5}) m / 2$.

The total energy of the rod system is expressed as follows

$$
\begin{gathered}
\mathbf{E}=\frac{1}{2}\left\{\xi_{2}^{2} \cos ^{2} \alpha_{12}+\left(\eta_{2}-\eta_{1}\right)^{2} \cos ^{2} \gamma_{12}+\frac{1}{4}\left(\eta_{2}-\eta_{1}\right)^{4}+\right. \\
+2 \xi_{2}\left(\eta_{2}-\eta_{1}\right) \cos \alpha_{12} \cos \gamma_{12}+\xi_{2}\left(\eta_{2}-\eta_{1}\right)^{2} \cos \alpha_{12}+\left(\eta_{2}-\eta_{1}\right)^{3} \cos \gamma_{12}+ \\
+2 m\left[\xi_{2}^{2} \cos ^{2} \alpha_{23}+\eta_{2}^{2} \cos ^{2} \gamma_{23}+\frac{1}{4} m^{2} \eta_{2}^{4}+2 \xi_{2} \eta_{2} \cos \alpha_{23} \cos \gamma_{23}-\right. \\
\left.\left.-m \xi_{2} \eta_{2}^{2} \cos \alpha_{23}-m \eta_{2}^{3} \cos \gamma_{23}\right]+n \xi_{2}^{2}\right\}-P_{1}^{*} \eta_{1}-P_{2}^{*} \eta_{2} .
\end{gathered}
$$

Where $P_{1}^{*}=P_{1} /(5 E F) ; P_{2}^{*}=P_{2} /(E F)$.

On the basis of the principle of stationarity of potential energy we obtain three total equations

$$
\begin{gathered}
\frac{\partial \mathbf{E}}{\partial \eta_{1}}=\frac{1}{2}\left(\eta_{1}-\eta_{2}\right)^{3}-\frac{3}{2}\left(\eta_{1}-\eta_{2}\right)^{2} \cos \gamma_{12}+\left(\eta_{1}-\eta_{2}\right) \cos ^{2} \gamma_{12}+ \\
+\xi_{2} \cos \alpha_{12}\left[\left(\eta_{1}-\eta_{2}\right)-\cos \gamma_{12}\right]-P_{1}^{*}=0 \\
\frac{\partial \mathbf{E}}{\partial \eta_{2}}=-\frac{1}{2}\left(\eta_{1}-\eta_{2}\right)^{3}+\frac{3}{2}\left(\eta_{1}-\eta_{2}\right)^{2} \cos \gamma_{12}- \\
-\left(\eta_{1}-\eta_{2}\right) \cos ^{2} \gamma_{12}-\xi_{2} \cos \alpha_{12}\left[\left(\eta_{1}-\eta_{2}\right)-\cos \gamma_{12}\right]+ \\
+m\left[m^{2} \eta_{2}^{3}-3 m \eta_{2}^{2} \cos \gamma_{23}+2 \eta_{2} \cos ^{2} \gamma_{23}+\right. \\
\left.-2 \xi_{2} \cos \alpha_{23}\left(m \eta_{2}-\cos \gamma_{23}\right)\right]-P_{2}^{*}=0 \\
\frac{\partial \mathbf{E}}{\partial \xi_{2}}=\frac{1}{2}\left(\eta_{1}-\eta_{2}\right)^{2} \cos \alpha_{12}-\left(\eta_{1}-\eta_{2}\right) \cos \alpha_{12} \cos \gamma_{12}- \\
-m \eta_{2}\left(m \eta_{2}-2 \cos \gamma_{23}\right) \cos \alpha_{23}+ \\
+\xi_{2}\left(\cos ^{2} \alpha_{12}+2 m \cos ^{2} \alpha_{23}+n\right)=0
\end{gathered}
$$

The result of addition of equations (8) and (9) can be presented as

$$
\begin{gathered}
m^{2} \eta_{2}^{3}-3 m \eta_{2}^{2} \cos \gamma_{23}+2 \eta_{2} \cos ^{2} \gamma_{23}- \\
-2 \xi_{2} \cos \alpha_{23}\left(m \eta_{2}-\cos \gamma_{23}\right)=\frac{1}{m}\left(P_{1}^{*}+P_{2}^{*}\right) .
\end{gathered}
$$

Taking into account the equation of connection (10), we find

$$
\xi_{2}=\zeta\left[-\frac{1}{2}\left(\eta_{1}-\eta_{2}\right)^{2} \cos \alpha_{12}+\left(\eta_{1}-\eta_{2}\right) \cos \alpha_{12} \cos \gamma_{12}+\right.
$$




$$
\left.+\mathrm{m} \eta_{2}\left(\mathrm{~m} \eta_{2}-2 \cos \gamma_{23}\right) \cos \alpha_{23}\right]
$$

Where $\zeta$ is

$$
\zeta=\left(\cos ^{2} \alpha_{12}+2 m \cos ^{2} \alpha_{23}+n\right)^{-1} .
$$

If we assume that the pentagonal ring of the two-tier dome is inextensible, then the equation (8) is

$$
P_{1}^{*}=\frac{1}{2}\left(\eta_{1}-\eta_{2}\right)^{3}-\frac{3}{2}\left(\eta_{1}-\eta_{2}\right)^{2} \cos \gamma_{12}+\left(\eta_{1}-\eta_{2}\right) \cos ^{2} \gamma_{12}
$$

The upper point of the curve $P_{1}^{*}=\left(\eta_{12}\right)$ we define believing

$$
\frac{\partial P_{1}^{*}}{\partial \eta_{12}}=0
$$

And $\eta_{12}=\eta_{1}-\eta_{2}$.

From this condition we find

$$
\eta_{12}^{2}-2 \eta_{12} \cos \gamma_{12}+\frac{2}{3} \cos ^{2} \gamma_{12}=0
$$

This follows

$$
\left(\eta_{12}\right)_{c r}=\left(1-\frac{\sqrt{3}}{3}\right) \cos \gamma_{12}
$$

In such a situation, we have a captivating result in its simplicity

$$
P_{1, c r}=\frac{5 \sqrt{3}}{9} E F \cos ^{3} \gamma_{12}
$$

For isolated from 320 fringe rod structure $m=0.85755$, and $n=1.1851$. The guides of the cosines of the terminals 1-2 and 2-3 have the following numerical values $\cos \alpha_{12}=$ 0.991950; $\cos \gamma_{12}=0.126591, \cos \alpha_{23}=0.375706 ; \cos \gamma_{23}=0.249624$.

The diagram $P_{1}^{*}\left(\eta_{12}\right)$ of equilibrium states of rod system, constructed on the basis of dependence (14), is shown in Fig. 2 black line. It is obvious that with increasing values $\eta_{12}$ system from the beginning behaves as a geometrically nonlinear

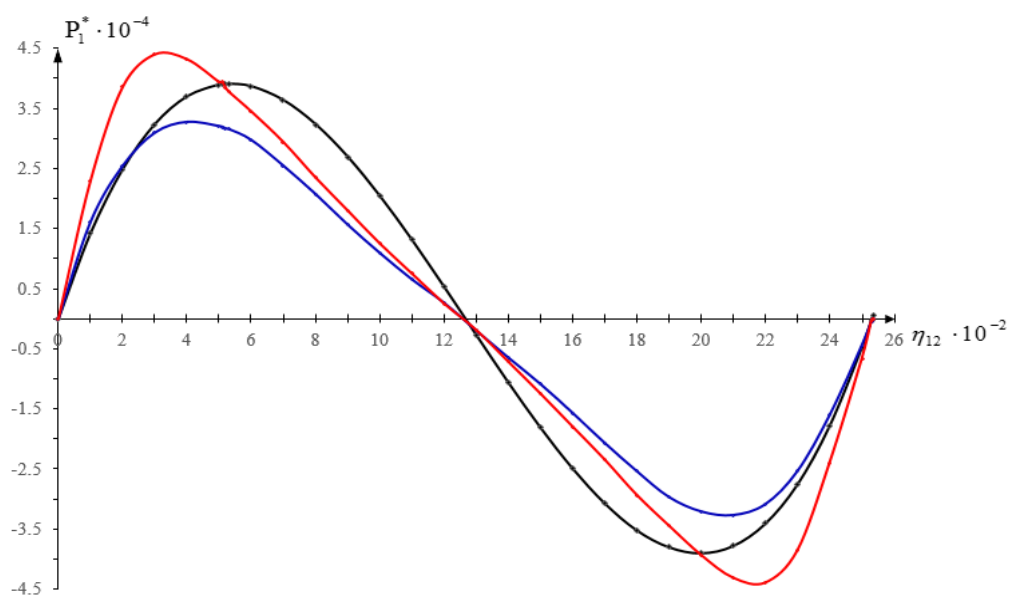

Fig. 2. Equilibrium state diagram for the two-tier part of the core structure in the form of a 320-granary 
Doing the same with respect to the equation (11), we present it as

$$
\hat{P}=\frac{1}{m}\left(P_{1}^{*}+P_{2}^{*}\right)=m^{2} \eta_{2}^{3}-3 m \eta_{2}^{2} \cos \gamma_{23}+2 \eta_{2} \cos ^{2} \gamma_{23} .
$$

Taking a derivative of $\hat{P}$ by parameter $\eta_{2}$ and equating it to zero, we obtain

$$
\frac{\partial \hat{P}}{\partial \eta_{2}}=3 m^{2} \eta_{2}^{2}-6 m \eta_{2} \cos \gamma_{23}-2 \cos ^{2} \gamma_{23}=0 .
$$

The smallest root of the square equation is

$$
\left(\eta_{2}\right)_{c r}=\left(1-\frac{\sqrt{3}}{3}\right) \Theta
$$

where $\Theta=\frac{1}{m} \cos \gamma_{23}$. Then

$$
\hat{P}_{c r}=\frac{2 \sqrt{3}}{9} \frac{1}{m} \cos ^{3} \gamma_{23} .
$$

Entering the coefficient $k=5 P_{2} / P_{1}$, taking into account the unevenness of the nodal load distribution, we find

$$
P_{1, c r}=\frac{10 \sqrt{3}}{9} \frac{E F}{1+k} \cos ^{3} \gamma_{23} .
$$

The other variant of the representation of equations (8) and (11) is obtained by excluding $\xi_{2}$ from them by means of the ratio (13) and writing these equations in a compact form as follows

$$
\begin{gathered}
P_{1}^{*}=\left(\eta_{12}-\right. \\
\left.+\cos \gamma_{12}\right)\left[\left(1-\zeta \cos ^{2} \alpha_{12}\right)\left(\frac{1}{2} \eta_{12}-\cos \gamma_{12}\right) \eta_{12}+\right. \\
\left.+\zeta \cos \alpha_{12} \cos \alpha_{23} m \eta_{2}\left(\mathrm{~m} \eta_{2}-2 \cos \gamma_{23}\right)\right] \\
\hat{P}=\left(1-2 m \zeta \cos ^{2} \alpha_{23}\right)\left(m^{2} \eta_{2}^{3}-3 m \eta_{2}^{2} \cos \gamma_{23}+2 \eta_{2} \cos ^{2} \gamma_{23}\right)+ \\
+\zeta \cos \alpha_{12} \cos \alpha_{23}\left(m \eta_{2}-\cos \gamma_{23}\right)\left(\eta_{12}-2 \cos \gamma_{12}\right) \eta_{12} .
\end{gathered}
$$

To fully determine the nature of the curves of the equilibrium states of the system, it is necessary in equation (23) to express $\hat{P}$ through $P_{1}^{*}$, and then, equating the right-hand side of the two equations, to determine the relationship between the two parameters of the angular displacements of the rods $\eta_{12}$ and $\eta_{2}$. In particular, we have

$$
\begin{gathered}
\left(\mathrm{m} \eta_{2}-2 \cos \gamma_{23}\right) \mathrm{m} \eta_{2}\left[\frac{1-2 m \zeta \cos ^{2} \alpha_{23}}{1+k}\left(\mathrm{~m} \eta_{2}-\cos \gamma_{23}\right)-\right. \\
\left.-\zeta \cos \alpha_{12} \cos \alpha_{23}\left(\eta_{12}-\cos \gamma_{12}\right)\right]= \\
=\left(\eta_{12}-2 \cos \gamma_{12}\right) \eta_{12}\left[\frac{1}{2}\left(1-\zeta \cos ^{2} \alpha_{12}\right)\left(\eta_{12}-\cos \gamma_{12}\right)-\right. \\
\left.-\frac{m \zeta \cos \alpha_{12} \cos \alpha_{23}}{1+k}\left(m \eta_{12}-\cos \gamma_{23}\right)\right] .
\end{gathered}
$$

In (26) it should now substitute the numerical values of the parameter $\eta_{12}$ in the interval of its changes, after which the least positive values are set by repeatedly solving the equations of the third degree with respect to the value of $\eta_{2}$, which should be introduced into the 
equilibrium equation (23). At the same time, the ratio of cargo areas for the case of uniformly distributed load, referred to the horizontal projection of the rod system, is such that $P_{2} / P_{1} \approx$ 1,34 . In this case, the calculation process leads to the dependence $P_{1}^{*}\left(\eta_{12}\right)$, shown in Fig.2 with a red line. As you can see, as a result of the inclusion in the work of compression of the rod elements of the pentagonal ring, the load level of the central node of the rod structure increases by $18 \%$ compared to the case when the ring is unsteady. The graph shows a blue line diagram $P_{1}^{*}\left(\eta_{12}\right)$ for the case of nodal loads of equal magnitude in both the central node 1 and five peripheral nodes 2 . Naturally, the load level of the central node is $16 \%$ lower in relation to its level, when the pentagonal ring is not included in the work of the rod structure of the two-tier dome.

\section{Conclusions}

The developed approach allows simplifying the calculations of rod systems of two-tier domes for stability under different types of loading. The approach is based on the known method of minimizing the functional of the total potential energy of deformation and allows to reduce the system of equations to simple formulas that can be used in engineering calculations.

The limit values of the load exceeding which leads to the loss of stability of the system and the appearance of bifurcation points are determined. The influence of the angle of inclination of the rods on the tendency to loss of stability is shown. Equilibrium state diagram for the two-tier part of the core structure in the form of a 320-granary is obtained and analyzed.

\section{References}

1. A.S. Volmir, Stability of deformable systems, Moscow, Science, 1967. P. 984

2. A. Zhuravlev, D. Zhuravlev, A. Melnik, MATEC Web of Conferences 106, 04023 (2017)

3. J. Błachut, Composite structures, 153, 746-754 (2016)

4. J. Błachut, Thin-Walled Structures, 47, 1429-1438 (2009)

5. Jiachuan Yan, Feng Qin, Zhenggang Cao, Feng Fan, Y.L. Mo, Engineering Structures 114, 158-170 (2016)

6. Jian Zhang, Benyi Zhu, Fang Wang, Wenxian Tang, Weibo Wang, Meng Zhang, ThinWalled Structures, 119, 296-303, (2017)

7. Zhejian Li, Wensu Chen, Hong Hao, International Journal of Impact Engineering, 115, 94-105 (2018)

8. E. Verwimp, T.Tysmans, M.Mollaert, S.Berg, Thin-Walled Structures, 94, 89-97 (2015)

9. J. Michael Rotter, Greig Mackenzie, Martin Lee, Structures 49, 2962-2977 (2012)

10. A.N. Beskopylny, E.E. Kadomtseva, G.P. Strelnikov, Y.A. Berdnik, IOP Conf. Ser.: Earth Environ. Sci., 90, 012064 (2017)

11. A.N. Beskopylny, E.E. Kadomtseva, G.P. Strelnikov IOP Conf. Ser.: Earth Environ. Sci., 90, 012017 (2017)

12. A.N. Beskopylny, A.A. Lyapin, MATEC Web of Conferences 117, 00018 (2017)

13. A.N. Beskopylny, A.A. Veremeenko, B.M. Yazyev, MATEC Web of Conferences 106, 04004 (2017)

14. A. Beskopylny, A. Veremeenko, et.al. MATEC Web of Conferences, 12902046 (2017)

15. A. Beskopylny, A. Lyapin, M. Kadomtsev, A. Veremeenko, MATEC Web of Conferences, 146, 02013 (2018) 